\title{
Constraining the physics of carbon crystallization through pulsations of a massive DAV BPM37093
}

\author{
Atsuko Nitta ${ }^{1},{ }^{2}$ S. O. Kepler ${ }^{3}$, André-Nicolas Chené ${ }^{1}$, D. Koester ${ }^{4}$, \\ J. L. Provencal ${ }^{5}$, S. J. Kleinmani ${ }^{1}$, D. J. Sullivan ${ }^{6}$, Paul Chote ${ }^{13}$, \\ Ramotholo Sefako ${ }^{7}$, Antonio Kanaan ${ }^{8}$, Alejandra Romero ${ }^{3}$, \\ Mariela Corti ${ }^{9,10}$, Mukremin Kilic ${ }^{11}$, M. H. Montgomery ${ }^{12}$ and \\ D. E. Winget ${ }^{12}$ \\ ${ }^{1}$ Gemini Observatory, Hilo, Hawaii, U.S.A. \\ email: anitta@gemini.edu \\ ${ }^{2}$ Subaru Telescope, NOAJ, Hilo, Hawaii, U.S.A. ${ }^{3}$ Universidade Federal do Rio Grande do Sul, \\ Porto Alegre, Rio Grande do Sul,Brazil \\ ${ }^{4}$ Universitat Kiel, Kiel, Germany \\ ${ }^{5}$ University of Delaware, Newark, DE, U.S.A. \\ ${ }^{6}$ Victoria University of Wellington, Wellington, New Zealand \\ ${ }^{7}$ South African Astronomical Observatory, P.O.Box 9, Capetown, 7935, South Africa \\ ${ }^{8}$ Universidade Federal de Santa Catarina, Florinopolis, Brazil \\ ${ }^{9}$ Instituto Argentino de Radioastronomía (CCT-La Plata, CONICET),Villa Elisa, Argentina \\ ${ }^{10}$ Universidad Nacional de La Plata, La Plata, Argentina \\ ${ }^{11}$ University of Oklahoma, Norman, OK, U.S.A. \\ ${ }^{12}$ University of Texas at Austin, Austin, TX, U.S.A. \\ ${ }^{13}$ University of Warwick, Coventry, CV47AL U.K.
}

\begin{abstract}
We are trying to reduce the largest uncertainties in using white dwarf stars as Galactic chronometers by understanding the details of carbon crystalliazation that currently result in a 1-2 Gyr uncertainty in the ages of the oldest white dwarf stars. We expect the coolest white dwarf stars to have crystallized interiors, but theory also predicts hotter white dwarf stars, if they are massive enough, will also have some core crystallization. BPM 37093 is the first discovered of only a handful of known massive white dwarf stars that are also pulsating DAV, or ZZ Ceti, variables. Our approach is to use the pulsations to constrain the core composition and amount of crystallization. Here we report our analysis of 4 hours of continuous time series spectroscopy of BPM 37093 with Gemini South combined with simultaneous time-series photometry from Mt. John (New Zealand), SAAO, PROMPT, and Complejo Astronomico El Leoncito (CASLEO, Argentina).
\end{abstract}

Keywords. stars: white dwarfs, stars: variables : other, stars: evolution

\section{Gemini Spectrum \& Model Fit}

We obtained approximately 4 hours of continuous time series spectra using GMOS-S at Gemini South. The integration time for individual exposure was 20 seconds, resulting in cycle time of about 35 seconds and total 380 frames of time series spectra. The data were taken in 1 arcsec or better seeing, photometric and dark sky condition. The combined 


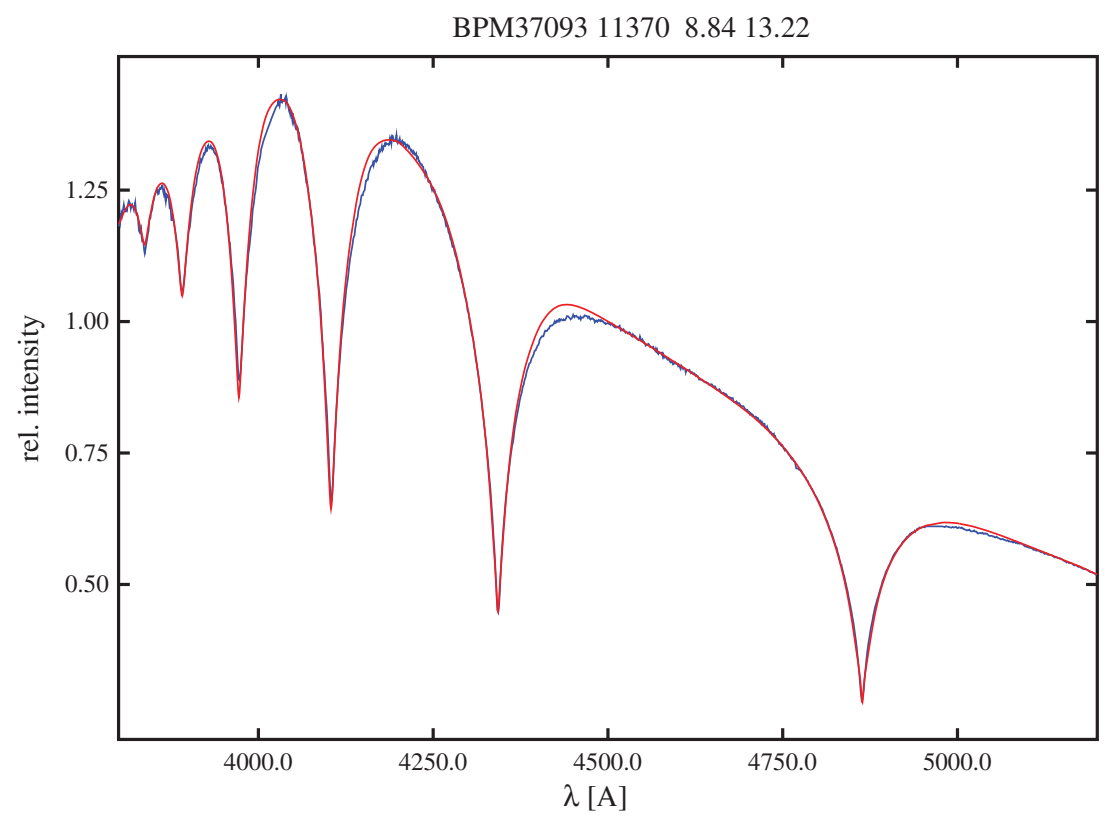

Figure 1. Gemini combined spectrum (red) and the 3rd model listed in the text (blue).

spectra which has equivalent to 7600 seconds integration time, has very high signal-tonoise which we used to fit with Koester models (Koester 2010). We have three equally good fits with different parameters, depending on slightly different treatment of the data and/or wavelength range used.

- $\mathrm{T}_{\text {eff }}=11,451 \mathrm{~K}, \log g=8.792$ using the combined spectrum up to $4950 \AA$ where there is a gap in the data corresponding to a gap between CCDs.

- $\mathrm{T}_{\text {eff }}=11,268 \mathrm{~K}, \log g=8.823$ using the combined spectrum up to $4950 \AA$ as above and cleaned up the high/low points due to the bad pixels which were not cleaned up during the reduction.

- $\mathrm{T}_{\text {eff }}=11,370 \mathrm{~K}, \log g=8.843$ using the combined spectrum up to $5200 \AA$ cleaned up the high/low points due to the bad pixels which were not cleaned up during the reduction, and adjusting the slope of the red part of the Gemini spectrum to make up for incomplete flux calibration.

The three results are consistent with previous determinations (Bergeron et al. 2004; Koester \& Allard 2000). The fits are statisitcally equivalent, but the 3rd fit which takes care of the shortcoming of the data reduction carried out likely is the best (Fig.1 )

The individual time series spectra show variations, mainly in flux, as one expects in a pulsator like BPM 37903. We did not detect any noticeable line profile variations originally seen the archived VLT spectra in $\mathrm{H} \beta$ and the higher order Balmer lines (Fig.2). We further found out that the calibration of the VLT spectra are not very accurate due to the wide slit used to acquire the data and the uncertainties are as large as the variation we were seeing. Therefore both observations are consistent with each other.

\section{Time Series Analysis}

Please see figures in Nitta et al.(2015) for the lightcurves, along with their Fourier Transform (FT) obtained by the Whole Earth Telescope (WET) and Gemini. 


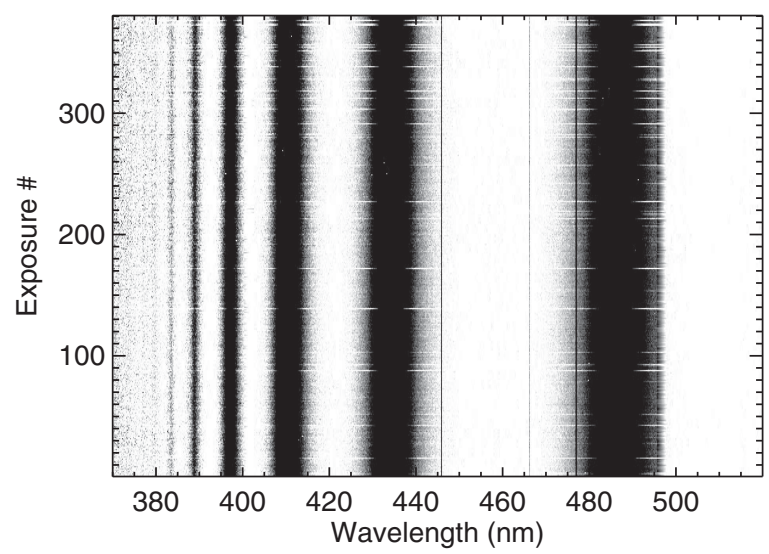

Figure 2. The combined Gemini time series spectra (7600 seconds integration) along with one of Detlev Koester's model (3rd model mentioned in the text) fit in red. Notice the high signal to noise of the observed spectrum.

Table 1. BPM 37093's Detected Pulsation Periods

\begin{tabular}{|c|c|c|c|c|c|c|}
\hline \multicolumn{2}{|c|}{$\ell$-value } & \multicolumn{3}{|c|}{$\mid$ Frequency $[\mu \mathrm{Hz}] \mid$ Period [sec] } & \multicolumn{2}{|c|}{ Amplitude [mma] | Note } \\
\hline | & & 1513.2 & I & 660.8 & 0.47 & No ID yet \\
\hline I & & 1560.0 & I & 641.0 & 2.9 & No ID yet \\
\hline | & 2 & 1570.6 & I & 636.7 & 1.74 & | \\
\hline | & 2 & 1578.5 & | & 633.5 & 1.31 & | \\
\hline | & & 1601.9 & I & 624.2 & 1.2 & No ID yet \\
\hline I & 1 & 1629.9 & I & 613.5 & 1.13 & I \\
\hline | & 2 & 1664.9 & I & 600.7 & 0.88 & | \\
\hline | & 2 & 1718.2 & I & 582.0 & 1.03 & I \\
\hline I & 2 & 1768.5 & I & 565.5 & 1.17 & $k=30, m=+1$ \\
\hline | & 2 & 1777.6 & I & 562.6 & 0.88 & $k=30, m=--1$ \\
\hline | & 2 & 1821.4 & 1 & 549.0 & 2.60 & I \\
\hline | & 2 & 1882.9 & I & 531.1 & 1.16 & I \\
\hline | & 2 & 1954.1 & I & 511.7 & 0.68 & I \\
\hline
\end{tabular}

We constructed a lightcurve by integrating the Gemini spectra in wavelength. We also derived $\mathrm{T}_{\text {eff }}$ and $\log g$ from each spectrum and call them "lightcurves" of $T_{\text {eff }}$ and $\log g$. The FT of WET data clearly shows two dominant modes, 549 second which has been observed in the past and 625 second mode which has not been observed before. We have checked via running Fourier transform of the WET data that this mode was stable throughout the WET run which are fortunate since BPM 37093 has shown amplitude modulations during WET runs in the past. We did not observe any definite signature of pulsation in the spectroscopic data. We have ruled out timing inaccuracy, data reduction introducing some artifacts etc. BPM 37093's low amplitude (1 to 2 mma as seen in the WET FT) meant that any pulsation signature is buried in the noise.

In the Table 1 , we listed all detected BPM 37093 pulsation modes from its discovery along with the $\ell$ l-value by Metcalfe, Montgomery \& Kanaan (2004). Please note that there are three modes that have not been ft by models yet. Our next step is to redo the 
model analysis to refine the crystallization mass fraction (currently estimated to be $37 \%$ up to $90 \%$ (Metcalfe, Montgomery \& Kanaan 2004; Brassard \& Fontaine 2005) perhaps using our newer models using the Horowitz crystallization prescription (Romero et al. 2013).

\section{Acknowledgements}

Based on observations obtained at the Gemini Observatory, which is operated by the Association of Universities for Research in Astronomy, Inc., under a cooperative agreement with the NSF on behalf of the Gemini partnership: the National Science Foundation (United States), the National Research Council (Canada), CONICYT (Chile), the Australian Research Council (Australia), Ministério da Ciência, Tecnologia e Inovação (Brazil) and Ministerio de Ciencia, Tecnología e Innovación Productiva (Argentina). This paper uses observations made at the South African Astronomical Observatory (SAAO).

\section{References}

Bergeron, P., Fontaine, G., Billres, M., Boudreault, S., \& Green, E. M., 2004, ApJ, 600, 404

Brassard, P. \& Fontaine, F., 2005, ApJ, 522, 572

Koester, D. 2010, Mem.S.A.It, 81, 921

Koester, D. \& Allard, N. F. 2000, Baltic Astronomy, 9, 119

Metcalfe, T. S., Montgomery, M. H. \& Kanaan, A., ApJ, 603, L133

Nitta, A. et al. 2015, Astronomical Society of the Pacific Conference Series, 493, 241

Romero, A. D., Kepler, S. O., Córsico, A. H., Althaus, L. G., \& Fraga, L. 2013, ApJ, 779, 58 